\title{
Chain dynamics on crossing the glass transition: non-equilibrium effects and recovery of the temperature-dependence of the structural relaxation
}

\author{
S. Arrese-Igor, ${ }^{1}$ A. Alegría, ${ }^{1,2}$ and J. Colmenero ${ }^{1,2,3}$ \\ ${ }^{1}$ Centro de Física de Materiales (MPC), Centro mixto CSIC-UPV/EHU, \\ Paseo Manuel Lardizabal 5, 20018 San Sebastián, Spain \\ ${ }^{2}$ Departamento de Física de Materiales UPV/EHU, Apartado 1072, 20080 San Sebastián, Spain \\ ${ }^{3}$ Donostia International Physics Center, Paseo Manuel Lardizabal 4, 20018 San Sebastián, Spain
}

(Dated: October 6, 2014)

\begin{abstract}
In this paper we report Thermally Stimulated Depolarization Current results on the chain and segmental dynamics of two monodisperse polyisoprenes accessing both dynamics at ultra-low frequency range and exploring the relationship between segmental and chain timescales when crossing the glass transition. In this range, we have recorded experimental evidence of non-equilibrium effects on the slowest chain mode dynamics. The non-equilibrium effects seem to occur simultaneously for both chain and $\alpha$-relaxation. Moreover, detailed analysis strongly indicates the recovery of an even T-dependence for the chain and $\alpha$-relaxation dynamics on crossing glass transition and in the glassy state. The obtained results can be understood taking into account the different temperature dependences of the length scales involved in the segmental and chain relaxations.
\end{abstract}

According to Rouse and reptation tube models ${ }^{1-3}$ for polymer chain dynamics, the characteristic time of the chain motion is defined in terms of the so-called friction coefficient and its temperature variation. Traditionally this coefficient has been identified with that controlling the segmental motions of the polymer chain leading to the expectation that both chain dynamics and the structural $\alpha$-relaxation have equal temperature(T)dependences. However, systematic tests of this assumption have proven that this is not the case and that the $\alpha$-relaxation shows a stronger $\mathrm{T}$-dependence than chain modes especially as temperature approaches the glass transition one, $T_{g} \cdot{ }^{4-7}$. Thermally stimulated depolarization current (TSDC) is a dielectric technique that has proven recently to be a very powerful tool allowing to study chain dynamics in detail at ultra-low frequencies ${ }^{8}$ and at relatively low temperatures close to $T_{g}$ - a range where chain end-to-end fluctuations detected by dielectric experiments have scarcely been explored. In this paper we report TSDC results on the chain and segmental dynamics of two monodisperse polyisoprenes accessing both dynamics at ultra-low frequency range and in this way exploring the relationship between segmental and chain timescales when approaching and crossing the glass transition. For low molecular weight polyisoprene (PI) non-equilibrium effects on the slowest chain mode are observed for the first time. Moreover, the detailed analysis of the results strongly indicates the recovery of an even T-dependence for the chain and $\alpha$-relaxation dynamics on crossing $T_{g}$ and deep in the glassy state.

Two monodisperse PI samples with molecular weights $M_{w}=10500 \mathrm{Da}$, PI10, and $M_{w}=2900 \mathrm{Da}, \mathrm{PI} 3$ were investigated. PI3 is below the entanglement regime, while PI10 is right at the transition between entangled and nonentangled systems. PI chain contains dipolar components parallel to the chain backbone yielding a net "end-toend" polarization vector. This allows to follow chain dynamics by dielectric relaxation techniques and as a consequence PI is a very well studied polymer by broadband dielectric spectroscopy (BDS) in particular. ${ }^{6,7,11,12} \mathrm{~A}$ TSDC experiment consist on: first, polarizing the sample at high temperature (where particular dipolar entities are mobile); then freezing-in the orientational polarization by cooling down the sample with the field on; switching off the field at low temperature; and finally recording the electric current due to the thermally stimulated release of the polarization during a subsequent linear heating. A peak in the electric current is obtained when the molecular mobility becomes high enough to randomize dipolar orientation. When a sample is fully polarized after the first and second steps, the measured current contains contributions from all the dielectrically active relaxations and the experiment is referred to as a global polarization TSDC experiment. TSDC technique, however, is unique in the sense that we can vary the temperature at which the field is switched on $\left(T_{o n}\right)$ or off $\left(T_{o f f}\right)$ during cooling to "remove" from the subsequent depolarization current curve the contribution from slow or fast relaxing entities, and in this way isolate the response of a certain process of interest. This is referred to as a partial polarization (PP) TSDC experiment. Here PP TSDC technique has been used to isolate the response of the $\alpha$-relaxation from that of the chain dynamics on the one hand, and to isolate the response of the slowest chain mode $p=1$ from faster $\alpha$-relaxation and higher order chain modes on the other. The later is based on the Rouse equation ${ }^{1}$ describing the end-to-end vector's $\vec{R}(t)$ correlation function as a discrete superposition of several mode components $<\vec{R}(t) \cdot \vec{R}(0)>\propto \sum_{p: o d d}^{N-1} \cot ^{2}\left(\frac{p \pi}{2 N}\right) \exp \left(-\frac{t}{\tau_{p}}\right)$ (where $N$ is the number of beads forming the chain, and $\tau_{p}=\tau_{1} / p^{2}$ is the relaxation time associated with the $p^{t h}$ mode). ${ }^{34}$ The slowest $p=1$ mode dominates the correlation function due to the $\sim 1 / p^{2}$ weighting factor. Moreover, it is 9 times slower than any other mode contributing to the polarization and consequently can be confidently isolated by PP TSDC experiments. ${ }^{8}$

Dielectric relaxation of the samples investigated is 


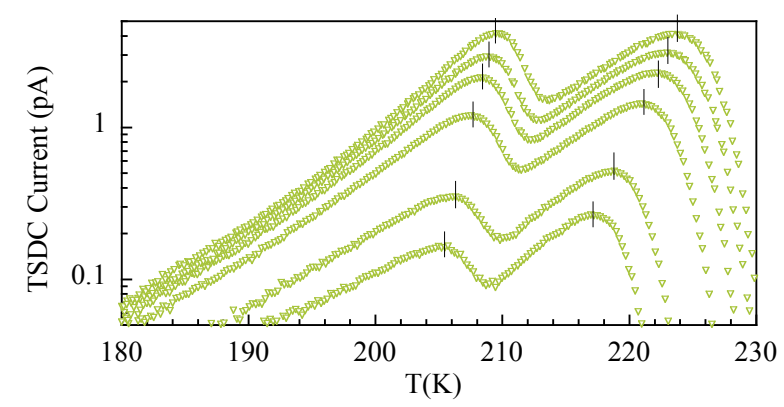

FIG. 1: Global depolarization current curves for PI10 recorded at 10, 7, 5, 3,1 and $0.5 \mathrm{~K} / \mathrm{min}$ (from top to bottom) heating rates. Vertical lines indicate maxima position.

typical of type-A polymers in general and PIs in particular. ${ }^{6,11,12}$ As an example, the global TSDC curves measured for PI10 at different heating rates are shown in figure 1 (for further experimental details please see supplementary information). The peak situated at lower temperature corresponds to the fast $\alpha$-relaxation whereas the peak at higher temperatures corresponds to the slower chain dynamics. As TSDC experiment is performed in non-isothermal condition, the response of the sample depends both on the heating rate and on the Tdependence of the characteristic times. Figure 2 shows with open squares the characteristic times for the chain and $\alpha$-relaxations calculated from the position of the maxima (vertical lines) in figure 1 (see supplementary information for further details). There, we also include relaxation times for segmental and chain dynamics $(\triangle)$ extracted by standard fitting procedures of isothermal BDS and time-domain relaxation data, showing good agreement.

As commented before, PP in TSDC allows to isolate specific contributions by applying a temperature cut-off in the exciting field. In particular, we have designed experimental protocols to isolate the response of the first $\mathrm{p}=1$ chain mode on the one hand ${ }^{8}$, and the response of the $\alpha$-relaxation on the other. Figure 3 shows the result of such experiments on PI10 (-o- for $\mathrm{p}=1$ chain mode and $-\nabla$ - for $\alpha$-relaxation). The outcome of a TSDC experiment can be numerically calculated assuming a general stretched-exponential -KohlrauschWilliams-Watts (KWW)- function for the relaxation of the polarization and using as inputs stretching exponent $\beta$ and T-dependent relaxation times (see supplementary information). For instance, the depolarization current curve corresponding to the $\mathrm{p}=1$ chain mode in PI10 can be calculated using as inputs: (i) the Williams-LandelFerry (WLF) ${ }^{13}$ law for the characteristic times extracted by fitting isothermal BDS measurements (solid line in figure 2a) and (ii) $\beta=1 .^{8}$ The so calculated current curve compares extremely well with the result of the PP experiment designed to isolate the $\mathrm{p}=1$ mode in PI10, strongly supporting the suitability of this procedure ${ }^{8}$ (see figure 3 ). What is more, the result of a calculation using the
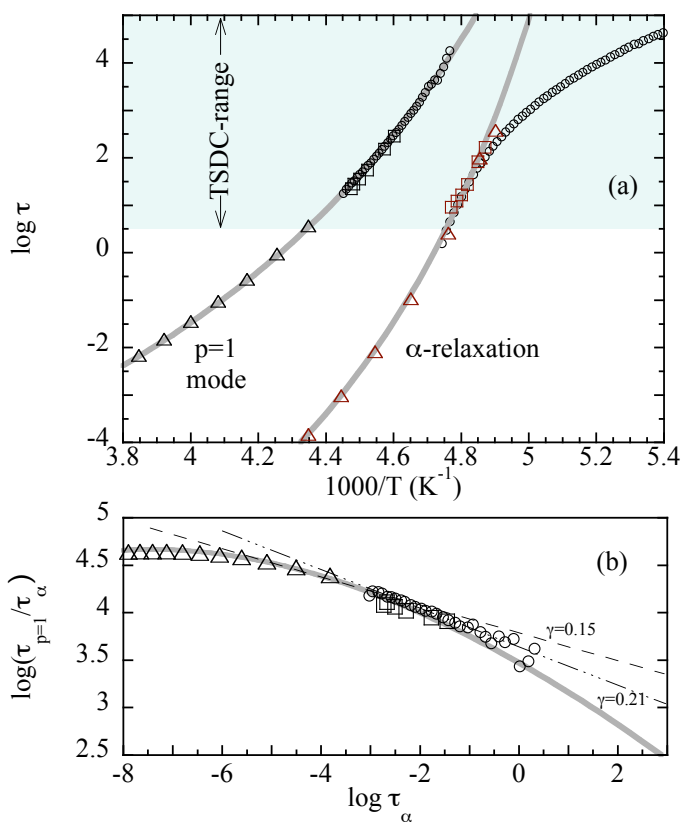

FIG. 2: Panel (a) $\tau(T)$ for the $\mathrm{p}=1$ chain mode and $\alpha$ relaxation of PI10 and (b) the ratio $\tau_{p=1} / \tau_{\alpha} .(\triangle)$ isothermal measurements; $(\square)$ variable rate global TSDC; (o) PP-TSDC. The lines in (a) are WLF and VFT law fits of the isothermal measurements and their ratio in (b). Dashed and dotted dashed lines in (b) show the power law description valid in limited ranges.

same WLF law as input but including this time up to $\mathrm{p}=9$ Rouse modes and the polarization conditions used in the real experiment is also indistinguishable from the experimental data and the calculated global $\mathrm{p}=1$ current curve. In addition, from the experimental TSDC data T-dependent characteristic times, $\tau_{T S D C}(T)$, can be obtained directly by analyzing the measured depolarization current as a function of temperature (see supplementary information). The so obtained times for the PP experiment of the $\mathrm{p}=1$ chain mode in PI10 using $\beta=1$ are included in figure $2 \mathrm{a}$ as circles. As it can be seen the agreement with the WLF law deduced from isothermal measurements is again very good, as already foreseen by the good concordance between calculated and experimental depolarization curves in figure 3. Noteworthy, this analysis of TSDC data for the $\mathrm{p}=1$ chain mode allows accessing relaxation times as high as $\sim 10^{5} \mathrm{~s}$ without noticing clear deviations from the behavior extrapolated from conventional isothermal experiments as seen in figure $2 \mathrm{a}$.

The fact that $\alpha$-relaxation shows a stronger $\mathrm{T}$ dependence than chain modes at $T \gtrsim T_{g}$ results very clear when low-frequency measurements of the chain dynamics by TSDC or time domain techniques are added to the conventional BDS frequency range. Differences in the T-variation of $\tau_{p=1}$ and $\tau_{\alpha}$ are further evidenced when plotting the ratio $\tau_{p=1} / \tau_{\alpha}$ as a function $\tau_{\alpha}$. In figure $2 \mathrm{~b}$ 
the dissimilar T-variation is obvious when approaching $T_{g}$ while it seems to vanish at much higher temperatures (where $\tau_{p=1} / \tau_{\alpha} \sim$ constant). Sokolov et al. ${ }^{9}$ approximated the ratio $\tau_{p=1} / \tau_{\alpha}$ close to $T_{g}$ by an effective power law $\tau_{p=1} / \tau_{\alpha} \propto\left(\tau_{\alpha}\right)^{-\gamma}$ and obtained $\gamma \simeq 0.15$ for PI. ${ }^{17}$ Our data clearly present some curvature so that the $\gamma$ value depends on the fitted $\tau_{\alpha}$ range, and varies for instance from $\gamma \simeq 0.15$ for $\log \tau_{\alpha}$ between -6 and -2 to $\gamma \simeq 0.21$ for $\log \tau_{\alpha}$ between -4 and 0 (see figure $2 \mathrm{~b}$ ). In this context, it has been reported that chain modes become non-exponential when their timescale approaches that of the structural $\alpha$-relaxation ${ }^{8}$. The origin of the disparate T-dependences of the chain and $\alpha$-relaxation, however, cannot be attributed to this effect. $\mathrm{p}=1$ chain mode for PI10 and higher molecular weight PI's are found to be exponential ${ }^{8}$ while their chain and $\alpha$-relaxation clearly show different T-dependences (see for example figure $2 \mathrm{~b}$ and reference ${ }^{9}$ ).

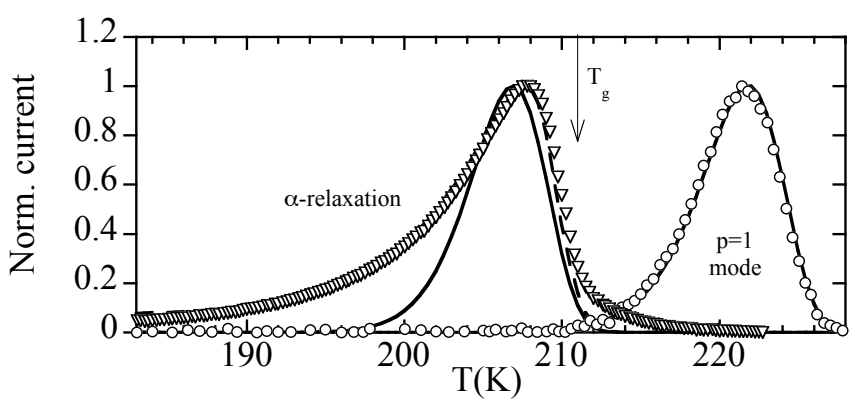

FIG. 3: PP current curves for PI10: (o) experimental $\mathrm{p}=1$ chain mode; $(\nabla)$ experimental $\alpha$-relaxation; solid lines calculations from VFT and WLF time laws in the metastable equilibrium; dashed line calculation from actual times extracted from the analysis of the experimental $\alpha$-relaxation current. Solid arrow represents calorimetric $T_{g}$ at the inflexion point of the DSC scan.

When the TSDC data of the $\alpha$-relaxation are analyzed clear non-equilibrium effects are manifested at $T<T_{g}$. The $\tau_{T S D C \alpha}(T)$ times calculated from the depolarization current curve of the $\alpha$-relaxation for PI10 using $\beta=0.48$ (determined from isothermal measurements) do not follow in the whole T-range the Vogel-FulcherTamman $(\mathrm{VFT})^{14}$ line determined by fitting isothermal measurements at metastable equilibrium condition (equilibrium from now on). Contrary, $\tau_{T S D C \alpha}(T)$ times show a crossover from an Arrhenius-like behavior deep in the glassy state $\left(T \ll T_{g}\right)$ towards a VFT-like one at higher temperatures. Accordingly, the TSDC curve calculated assuming the VFT line in equilibrium and $\beta=0.48$, clearly fails in describing experimentally recorded current (see solid line in figure 3), evidencing that during the experiment the characteristic times of the $\alpha$-relaxation at $T \ll T_{g}$ do not follow the VFT law assumed in this calculation. This is not an unexpected result, as in practice, within the experimental timescale it is not possible to infinitely undercool the melt, and eventually the system will go out of the supercooled metastable equilibrium into the glassy state when the $\alpha$-relaxation time reaches values in the range 1-100 $\mathrm{s}$. The effect of crossing the glass transition on the $\alpha$-relaxation timescale is well docummented ( $\mathrm{see}^{18}$ and references therein). A crossover from VFT to a milder Arrhenius-like T-dependence was reported by numerous studies and several experimental techniques ${ }^{20-28}$. The question of whether this crossover only occurs for not fully equilibrated glasses is a topic of intense research activity ${ }^{29}$ but out of the scope of this work.

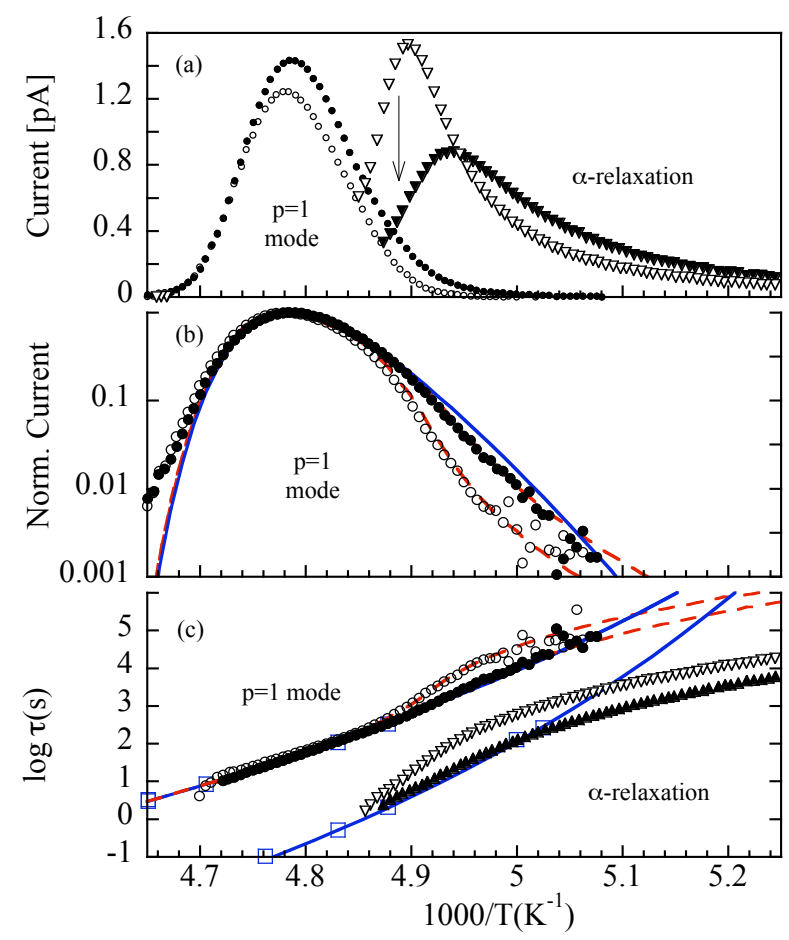

FIG. 4: PP-TSDC currents and $\tau_{T S D C}$ times for $\mathrm{p}=1$ chain mode (circles) and $\alpha$-relaxation (triangles) with (empty symbols) and without (solid symbols) aging. Solid lines represent VFT or WLF laws in equilibrium in (c) or the currents calculated on their basis in (b). Dashed lines represent adhoc times for $\mathrm{p}=1$ chain mode (see text) in (c) and the currents calculated on their basis in (b). Squares in (c) represent characteristic times determined at equilibrium condition by isothermal measurements. Solid arrow in (a) represents calorimetric $T_{g}$ at the inflexion point of the DSC scan.

At this point the question arises if similar nonequilibrium effects on crossing the glass transition can be observed for the chain dynamics, something that to our knowledge has never been directly measured before for the slowest chain mode. Even in the ultralow frequency range accessed by TSDC $\left(\lesssim 10^{5} \mathrm{~s}\right)$, the characteristic times for the $\mathrm{p}=1$ chain mode of PI10 do not show significant deviations from the equilibrium behavior extrapolated from isothermal measurements. Taking the crossover of the $\alpha$-relaxation as a reference, it seems that the chain relaxation times where such non-equilibrium ef- 
fects would be expected exceed the accessible time range by TSDC for the PI10 sample considered so far. Since by lowering molecular weight chain dynamics becomes significantly faster, a lower molecular weight PI sample (PI3) was investigated using the same approach. Figure 4 shows the result of PP experiments designed to isolate the response of the $\mathrm{p}=1$ mode and the $\alpha$-relaxation of PI3 (panels a and b) together with the characteristic times extracted from these data (panel c). When analyzing these depolarization curves, $\beta=0.48$ was assumed for the $\alpha$ relaxation whereas for the $\mathrm{p}=1$ chain mode $\beta=0.87$ was used according to the results in reference ${ }^{8}$. Contrary to the PI10 case, when the experimental data for $\mathrm{p}=1$ chain mode are compared with the expectation for a mere extrapolation of the equilibrium behavior (solid line in figure $4 \mathrm{~b}$ ) some differences are observed in the low temperature side, namely below $T_{g}$. Trying to confirm that the observed deviations are truly related to non-equilibrium effects, new experiments involving annealing below $T_{g}$ were performed. Since annealing the glassy material below $T_{g}$ results in a structural recovery (aging) approaching the supercooled metastable equilibrium state, the final glassy state obtained after cooling the aged sample should have a reduced mobility with the result of a more dramatic crossover phenomena on approaching $T_{g}{ }^{19}$. The experimental data obtained under these conditions (open symbols in figure 4) confirm more prominent effects not only on the $\alpha$-relaxation but, more importantly, also on the $\mathrm{p}=1$ chain mode. The difference between the depolarization curve predicted for a $p=1$ mode relaxation following the equilibrium WLF law in the whole temperature range (solid line) and the experimental data of the aged sample is now very obvious. Even more, when the relaxation times for these annealed samples are evaluated the crossover phenomena is clearly visible also for the slowest chain mode, mainly at the lowest temperatures. Results in figure 4 prove the emergence of non-equilibrium effects on the slowest chain mode when crossing the glass transition. Similar to the phenomenology observed for the $\alpha$-relaxation, at $T \lesssim T_{g}$ the $\mathrm{p}=1$ chain mode deviates from the extrapolation of the WLF law deduced from higher temperature experiments covering the more conventional frequency range, whereas at $T \gtrsim T_{g}$ nicely follows it. Furthermore, this crossover is more dramatically manifested when the polymer is annealed below $T_{g}$.

As already commented, the connection between the polymer chain dynamics and the $\alpha$-relaxation has been investigated in detail, in particular the problem of the thermorheological complexity, referring to the different T-dependences of these two dynamics. It has been found that whereas at temperatures well above $T_{g}$ both follow approximately the same T-dependence, there is a clear decoupling on approaching $T_{g}$. The current understanding of the physics behind this different evolution with temperature remains unclear ${ }^{17,30-33}$. In general different explanations put the accent on: (i) the spatially heterogeneous dynamics of the $\alpha$-relaxation in contrast to an averaged effect of nanoscale heterogeneities on the chain relaxation; or (ii) the very distinct $T$-evolution of the length scales involved on the chain dynamics and the $\alpha$-relaxation, which for the case of the later rapidly increases as temperature approaches $T_{g}$. The results presented herein indicate that around $T_{g}$ chain dynamics and $\alpha$-relaxation both present a similar crossover and alike $\mathrm{T}$ dependences below $T_{g}$. A detailed analysis of this range never explored before should shed new light on the issue.

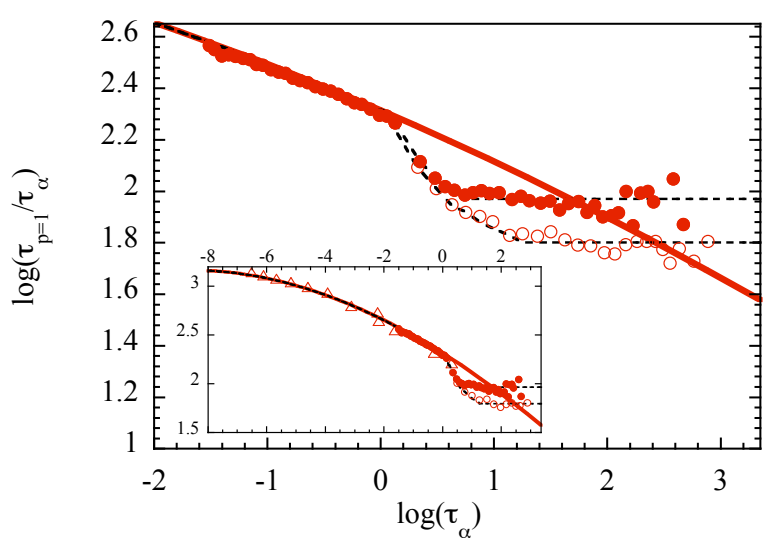

FIG. 5: $\mathrm{p}=1$ chain mode and $\alpha$-relaxation characteristic times ratio: solid lines VFT and WLF laws obtained fitting isothermal measurements; (•) non-aged PI3; (०) well-aged PI3; dashed lines ad-hoc $\tau_{p=1}$ times (see text). The inset represents the same in a wider scale including also isothermal data $(\triangle)$.

Figure 5 shows the $\tau_{p=1} / \tau_{\alpha}$ values for PI3 obtained by TSDC measurements during continuous heating through the $T_{g}$ range as well as those obtained by isothermal measurements at equilibrium. For values of $\tau_{\alpha}$ lower than a few seconds $\left(T>T_{g}\right)$ the $\tau_{p=1} / \tau_{\alpha}$ ratio conforms with the extrapolated equilibrium line. On the contrary the appearance of the data in the "out of equilibrium" range, $\tau_{\alpha}>1 \mathrm{~s}$, changes rather dramatically. There, it becomes evident that the ratio between the chain and the $\alpha$-relaxation timescales in both non-annealed and wellannealed samples does not longer follow the monotonous variation expected from the extrapolation of equilibrium measurements. Data look nearly constant at the lowest temperatures and cross over abruptly towards the equilibrium behavior at higher temperatures. The flattening of the data at the lowest temperatures is indicative of a trend to fully recover the pairing of the $\mathrm{T}$ dependences of both dynamical processes deep in the glassy state. This idea is further sustained by the fact that assuming ad-hoc $\tau_{p=1}(T)$ times (dashed lines in figure $4 \mathrm{c}$ ) yield calculated (using $\beta=0.87$ ) currents for $\mathrm{p}=1$ chain mode that describe very well the experimentally measured ones, in both non-annealed and well-annealed PI3 (see dashed lines in figure 4b). These ad-hoc times for $\mathrm{p}=1$ chain mode were constructed starting from experimental $\tau_{T S D C \alpha}(T)$ times and assuming the $\tau_{p=1} / \tau_{\alpha}$ 
ratios indicated by dashed lines in figure 5 , that is, the same T-dependence below $T_{g}$ and then smooth crossover to equilibrium values.

The restoring of the same T-dependence of the chain and segmental dynamics well below $T_{g}$ is an expected result when the thermorheological complexity is explained as originated by the very distinct $\mathrm{T}$-dependence of the length scales involved ${ }^{30}$. The argument of this interpretation is based on the general idea that the length scale characteristic of the $\alpha$-relaxation is T-dependent and increases rather dramatically on approaching $T_{g}$, where it reaches a value close to the Kuhn length. Contrary, the typical length scale controlling chain fluctuations is of the order of the radius of gyration which remains essentially $\mathrm{T}$-independent. This would explain the stronger T-dependence of $\tau_{\alpha}$ when approaching $T_{g}$ and the nearly constant $\tau_{p=1} / \tau_{\alpha}$ ratio at high temperatures (see inset of figure 5) as a result of the rather weak T-dependence of both length scales in this range. With these same arguments in mind, when cooling below $T_{g}$ the $\alpha$-relaxation length scale cannot longer increase and consequently both dynamical processes are expected to follow a similar T-dependence. Similarly, regarding the role of dynamic heterogeneities on the emergence of thermorehological complexity, the present results would put the accent on the T-evolution of their amplitude or length scale (which would arrest or freeze upon crossing $T_{g}$ ) rather than on their mere presence.

\section{Acknowledgments}

We acknowledge the support of the following research projects MAT2012-31088 supported by the Spanish Ministry "Ministerio de Economía y Competitividad" and IT-654-13 supported by the Basque Government.

\section{SUPPORTING INFORMATION AVAILABLE}

Experimental and data analysis details. This material is available free of charge via the Internet at http://pubs.acs.org.
1 Rouse, E.P. J. Chem. Phys.; 1953; 21, 1272.

2 Doi, M.; Edwards, S.F. The Theory of Polymer Dynamics; Oxford Science Publications. ISBN-0-19-852033-6.

3 de Gennes, P. G. J. Chem. Phys. 1971, 55, 572.

4 Plazek, D. J. J. Phys. Chem. 1965, 69, 3480.

5 Plazek, D. J.; O'Rourke, V. M. J. Polym. Sci., Part A-2. 1971, 9, 209.

6 Boese, D.; Kermer, F. Macromolecules 1990, 23, 829-835.

7 Nicolai, T.; Floudas, G. Macromolecules 1998, 31, 2578.

8 Arrese-Igor, S; Alegría A.; Colmenero, J. Phys. Rev. Lett. 2014,113, 078302.

9 Sokolov, A. P.; Hayashi, Y. J. Non-Cryst. Solids 2007, 353, 3838-3844.

10 Stockmaye rW. H. Pure Appl. Chem. 1967, 15, 539.

11 Matsumiya,Y.; Kumazawa, K.; Nagao, M.; Urakawa, O.; Watanabe, H. Macromolecules 2013, 46, 6067-6080.

12 Watanabe, H.; Matsumiya,Y., Osaki, K.; Yao, M. L. Macromolecules 1998, 31, 7528-7545.

13 M. L. Williams, R. F. Landel, and J. D. Ferry, J. Am. Chem. Soc. 1995, 77, 3701.

14 H. Vogel, J Phys. Zeit. 1921, 22, 645.; G. S. Fulcher, J. Am. Ceram. Soc. 1925, 8, 339.; G. Tammann and W. Hesse, Z. Anorg. Allg. Chem.,1926, 165, 254

15 Braünlich, P. Thermally Stimulated Relaxations in Solids, Topics in Applied Physics; Ed., Springer-Verlag:New York,

16 Sessler, G. M. Electrects, Topics in Applied Physics; Ed., Springer-Verlag:New York, 1980; Vol. 33, ISBN-978-3540-70750-9.

17 Sokolov, A. P.; Schweizer K. S. Phys. Rev. Lett. 2009, 102, 248301.

18 Cangialosi, D. J. Phys.: Condens. Matter 2014, 26, 153101.
19 Boucher, V. M.; Cangialosi, D.; Alegría, A.; Colmenero, J. Phys. Rev. E 2012, 86, 041501.

20 Zhao, J. K.; Simon, S. L.;McKenna, G. B. Nature Commun. 2013, 4, 1783.

21 Simon, S. L.; Sobieski, J. W.; Plazek, D. J. Polymer 2001, 42, 2555-2567.

22 Boucher, V. M.; Cangialosi, D.; Alegría, A.; Colmenero, J. Macromolecules 2011, 44, 8333-8342.

23 Hwang, Y.; Inoue, T.; Wagner, P. A., Ediger, M. D. J. Polym. Sci. B 2000, 38, 68-79.

24 Thurau, C. T.; Ediger, M. D. J. Chem. Phys. 2003, 118, 1996-2004.

25 Casalini, R.; Roland, C. M. Phys. Rev. Lett. 2009, 102, 035701.

26 Adrhanowicz, K.; Paluch, M.; Ngai, K. L. J. Phys.: Condens. Matter 2010, 22, 125902.

27 Alegría. A.; E. Guerrica-Echevarra; Goitiandia, L.; I. Telleria; Colmenero, J. Macromolecules 1995, 28, 1516-1527.

28 Alegría. A.; Goitiandia, L.; Colmenero, J. J. Polym. Sci. B: Polym. Phys 2000, 38, 2105-2113.

29 Cangialosi, D.; Boucher, V. M.; Alegría, A.; Colmenero, J. Phys. Rev. Lett. 2013, 111, 095701.

30 Schönhals, A. Macromolecules 1993, 26, 1309-1312.

31 Ngai, K. L., Casalini, R.,Roland, C. M.Macromolecules 2005, 38, 4363.

32 Ilan, B.; Loring, R. F. Macromolecules 1999, 32, 949.

33 Ngai, K. L., Roland, C. M. J. Chem. Phys. 2013, 139, 036101.

34 The Rouse equation for the end-to-end vector also applies for entangled polymers within the reptation theory and its extensions with a different physical meaning for $\tau_{p=1}$. 